\title{
Long-term maintenance of shuttlebox avoidance behavior before and after septal lesions
}

\author{
ALEX POPLAWSKY \\ Bloomsburg State College, Bloomsburg, Pennsylvania 17815
}

\begin{abstract}
Twenty-four rats were given 1,800 trials in a two-way avoidance chamber. Rats that avoided shock on more than $50 \%$ of the trials were categorized as high avoiders, while the others were categorized as low avoiders. Half the animals in each group received septal lesions, while the remaining animals received control operations. These four groups of animals were then observed postoperatively for another 1,800 trials of signaled avoidance. Preoperative low avoiders exhibited a large postoperative increase in avoidances, while high avoiders continued to avoid shock after septal lesions. Animals with septal lesions maintained a high level of avoidance throughout the postoperative testing period. Intertrial crossing had an initial postoperative increase in animals with lesions, which gradually decreased over days. Septal lesions interfered with the suppression of freezing behavior, thereby facilitating the performance of low avoiders without changing high-avoider performance. Comparisons of septal lesions and scopolamine were discussed.
\end{abstract}

It is well established that septal lesions facilitate the acquisition of a two-way active avoidance problem (for a review, see Fried, 1972). Changes in activity, that is, increased activity following septal lesions, as measured by intertrial crossing (Schwartzbaum, Green, Beatty, \& Thompson, 1967) and reduced freezing responses folowing septal lesion (Kenyon \& Krieckhaus, 1965; Schwartzbaum et al., 1967), have been implicated as possible causes for this effect. For example, Schwartzbaum et al. (1967) found a high relationship between learning an avoidance response and unpunished intertrial crossings. However, further studies (Johnson, Poplawsky, Bieliauskas, \& Liebert, 1972; Kenyon \& Krieckhaus, 1965; Meyer, Johnson, \& Vaughan, 1970) demonstrated that rats with septal damage still exhibited a facilitated avoidance response with punished intertrial crossings. Meyer et al. (1970) also concluded that the avoidance facilitation effect following septal lesions could not be explained by changes in intertrial crossings alone. In a recent study comparing avoidance performance with punished or unpunished intertrial crossings, Blatt (1976) found that animals with septal damage avoided more shocks than control animals only when intertrial crossings were punished. He concluded that septal lesions interfered with the suppression of the ineffective defense reaction of freezing, thereby facilitating avoidance performance when a passive avoidance contingency (punished intertrial crossings) was added to the active avoidance contingency.

Shock-induced suppression of running behavior inhibits the acquisition of the avoidance response (Bolles, 1970), causing many animals not to learn to avoid shock. An animal that is a poor avoider after many trials of training is usually a consistent escaper. Since escape is the primary way of coping with the shock, this further reduced the probability of the animals learning the avoidance contingency (Carlton, 1969). McCleary (1961) proposed that septal lesions resulted in a failure to suppress responses that have a high probability of occurrence, therefore low-probability responses would become less probable following a septal lesion. It would then follow that animals that were experienced low avoiders should show no improvement in avoidances following septal lesions. A number of studies have investigated the effects of previous avoidance experience on avoidance responding after septal damage. Worsham and Hamilton (1973) showed that rats with 60 trials of preoperative training still exhibited an increase in avoidances for another 60 trials after a septal lesion. Using a leverpress avoidance, Hedges, Van Atta, and Thomas (1975) found that septal lesions facilitated avoidance responding in rats with 1,000 trials of consistent escape responding with avoidance of shock on less than $5 \%$ of the trials.

The purpose of this experiment was to investigate whether septal lesions resulted in a disinhibition of a high probability of response (McCleary, 1961), or interfered with the suppression of an ineffective defense reaction such as freezing (Blatt, 1976). This was accomplished by categorizing the animals as high or low avoiders after 1,800 trials of avoidance-training experience. If septal lesions interfered with suppression of a high-probability response, then low-probability responses should become less probable. This would cause a decrease or no change in avoidance performance in low avoiders (since freezing would become more probable), and an increase in avoidance performance in high avoiders (since running would become more probable). 
However, if septal lesions interfered with the suppression of the ineffective defense reaction of freezing, which is a high-probability response for a low avoider and a low-probability response for a high avoider, low avoiders should have a large increase in avoidance, while high avoiders should only have a slight increase. The relationship between avoidances and intertrial crossings over extended training was also examined.

\section{METHOD}

\section{Animals}

Twenty-four adult male albino rats of the Sprague-Dawley strain (Camm Research, New Jersey), weighing 270-300 g at the beginning of the experiment were used. Each rat was housed individually, with free access to food and water for the duration of the study. Two rats died during the experiment, and another rat's data were eliminated because of an inappropriate avoidance response (standing upright on one shock grid).

\section{Apparatus}

The test apparatus was an automated dual-compartment shuttlebox (Lehigh Valley Electronics, Model 146-04) contained within a sound-attenuated cubicle (Lehigh Valley Electronics, Model 132-06). The chamber's dimensions were $27.3 \mathrm{~cm}$ height $\times 21.6 \mathrm{~cm}$ width $\times 48.3 \mathrm{~cm}$ length. A $28-\mathrm{V}$ dc light bulb was mounted $14 \mathrm{~cm}$ above the shock grids on the center far wall of each compartment. A barrier with a circular opening $(6.3 \mathrm{~cm}$ diam) separated the two compartments. The toggle floor reported the animals' location during and between trials. Shock was delivered to the grid floor of each compartment by a Lehigh Valley Electronics shock scrambler (Model SC-902). Sessions were controlled automatically by solid state circuitry (Coulbourn Electronics).

\section{Procedure}

Preoperative procedure. Each rat was placed in the test chamber for $90 \mathrm{~min}$ for each of 3 days before avoidance conditioning. Both cue lights, later to be the conditioned stimulus (CS), were presented at varying time intervals, averaging $30 \mathrm{sec}$ (a random-time schedule of $30 \mathrm{sec}$ with a minimum interval of $7.5 \mathrm{sec}$ ), and remained on until the animal crossed into the other compartment. Avoidance testing began on the next day for 30 daily sessions of 60 trials a session. A trial began with presentation of the CS (cue lights on) approximately $30 \mathrm{sec}$ after each rat was placed in the dark shuttlebox. If the rat did not cross into the other half of the chamber within $10 \mathrm{sec}$ of the onset of the cue lights, a continuous shock of $1 \mathrm{~mA}$ was applied to the grid floor of that side of the apparatus. An avoidance response (compartment crossing within $10 \mathrm{sec}$ of light onset) terminated the lights and the shock. The intertrial interval was determined by the random-time schedule of $30 \mathrm{sec}$. Responses made during the intertrial interval were not punished. Following each session, the avoidance latency, escape latency, and the number of escapes, avoidances, and intertrial crossings were recorded.

Surgical procedure. After preoperative testing, the rats were divided into two groups based on the average number of avoidances in the last 10 preoperative days. Rats that successfully avoided shock on more than $50 \%$ of the trials were categorized as high avoiders, the others as low avoiders. Half of the animals in each group either received septal lesions or were operated controls. The 21 animals were therefore divided into four groups: low avoiders receiving septal lesions (Group LS), $n=6$; high avoiders receiving septal lesions (Group HS), $n=5$; low avoiders receiving no lesions (Group $L N), n=5$; and high avoiders receiving no lesions (Group HN), $n=5$.

The animals were anesthetized with ethyl ether and placed in a Kopf stereotaxic instrument. Septal lesions were accomplished by a temperature-controlled radio-frequency lesion generator (Radionics, Inc., RFG-4), using a temperature monitoring

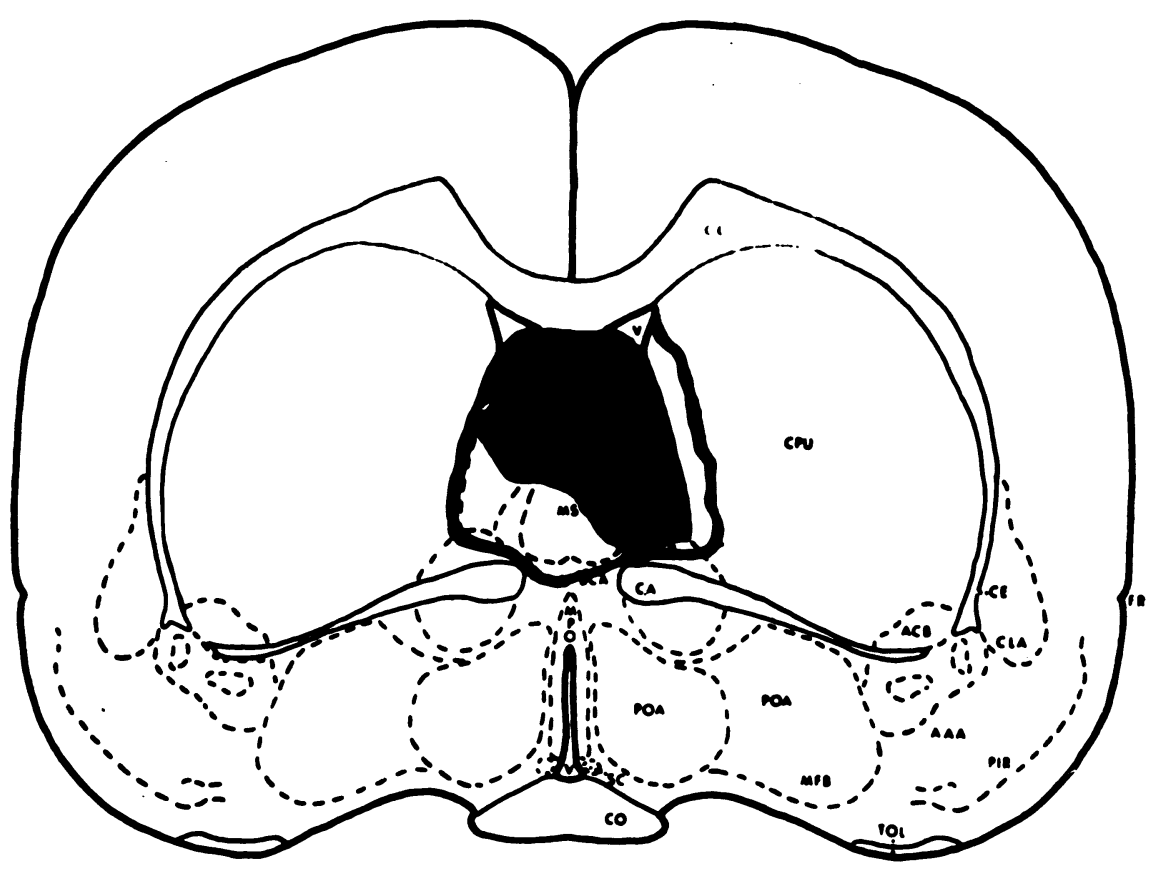

Figure 1. Reconstruction showing the size of the septal lesions in the experimental groups. Solid black area shows the damage common to 10 of the 11 animals with septal lesions. Black outline represents the largest lesion. 
electrode (Radionics, Inc.). Current adjustments were made until the temperature of the electrode tip stabilized at $60^{\circ} \mathrm{C}$ for a total of $60 \mathrm{sec}$. The tip of the electrode was placed stereotaxically in accord with modified de Groot (1959) coordinates. The modification was to adjust the head such that lambda and bregma were on the same horizontal plane and to adjust the de Groot coordinates accordingly. The coordinates for septal lesions were: $10.5 \mathrm{~mm}$ anterior to the interaural line, $5.0 \mathrm{~mm}$ below the dura at the midline. The operated controls underwent the same surgical procedure, except the electrode tip was lowered $2.0 \mathrm{~mm}$ below the dura with no current.

Postoperative and histological procedures. After $24 \mathrm{~h}$ recovery, each animal was tested for 30 sessions, using the preoperative procedures earlier described. After 30 sessions, all animals were deeply anesthetized with ether and perfused intracardially with $.9 \%$ physiological saline $(.9 \% \mathrm{NaCl})$ followed by $10 \%$ Formalin solution. Brains were extracted, and frozen brain sections were made every $80 \mu$. To determine the location and extent of the lesion, every second section through the lesion was mounted on a slide, stained with cresyl violet, and examined microscopically. Every third section was projected on appropriate Pellegrino and Cushman (1967) atlasreference coronal planes, and reconstructions of the lesions were used to determine what structures were damaged. Percent of damage was determined by projecting these coronal sections on a grid of 1-mm squares.

\section{RESULTS}

\section{Histology}

There were no gross differences between lesions in Groups LS and HS. The septal lesions generally extended from the genu of the corpus callosum to the columns of the fornix (anterior-posterior) and from the corpus callosum to the anterior commissure (dorsal-ventral). Figure 1 presents the largest lesion and the common area of destruction for all animals. There was a 45\%-50\% sparing of the medial septum in two animals in each lesioned group. One animal in Group LS had a lesion more ventrally placed than the other animals, destroying the medial septum, the ventral lateral septum, and damaging the bed nuclei of the anterior commissure and the anterior commissure.

\section{Behavior}

A 4 (groups) by 3 (days) mixed design analysis of variance was used to evaluate the habituation (preavoidance training) data. There was a significant increase in the amount of time the animals left the light on over days $(68,74,78 \mathrm{~min}$ out of $90 \mathrm{~min}$, respectively) during the habituation period $[\mathrm{F}(2,34)=10.45, \mathrm{p}<.01]$. The animals therefore made fewer intercompartment crossings over days $(39,30,24$, respectively) $[\mathrm{F}(2,34)=$ $13.52, \mathrm{p}<.01]$; an average of 4.5 crossings occurred within $10 \mathrm{sec}$ of light onset. There were no significant differences among groups.

A 4 (groups) by 15 (2-day blocks) by 2 (surgery) mixed design analysis of variance was used to evaluate changes in number of avoidances, intertrial crossings, avoidance latencies, and escape latencies. The days factor was analyzed in blocks of two.

Figure 2 shows the mean number of avoidances out of a possible 60 for the four groups of animals for preoperative and postoperative blocks of 2 days. There was a significant groups effect indicating that Groups HS and $\mathrm{HN}$ were the highest avoiders, Group LN the lowest avoiders, and Group LS the intermediate avoiders $F(3,17)=46.75, p<.001]$. The day effect showed that animals exhibited the lowest number of avoidances during the first block of avoidance sessions, with a mean of 26.9 avoidances; the other avoidance sessions averaged 33.4 avoidances $[F(14,238)=4.85, p<.001]$. A significant Days by Group effect showed that avoidance decreased in the first third of the avoidance
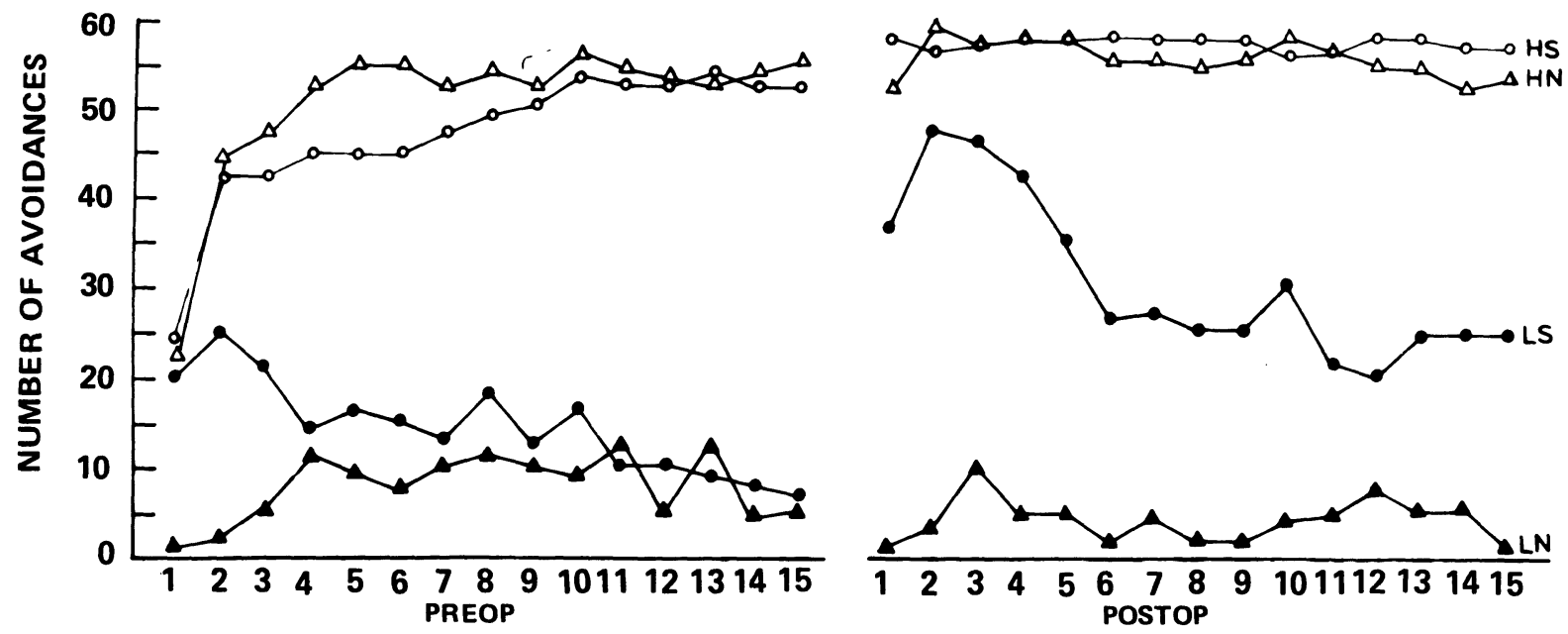

\section{BLOCKS OF 2 DAYS}

Figure 2. Mean number of avoidances for low avoiders (LS) and high avoiders (HS) with septal lesions and low avoiders (LN) and high avoiders (HN) with control operations for each 60-trial session averaged in blocks of 2 days before and after surgery. 
sessions for the low septal group while the other groups were still increasing $[F(42,238)=6.13, p<.001]$. A significant surgery effect indicated that the animals exhibited an overall postoperative increase in avoidances from a preoperative mean of 29.3 avoidances to a postoperative mean of 36.7 avoidances $[F(1,17)=11.81$, $\mathrm{p}<.005]$. A significant Groups by Surgery interaction showed that different groups of animals had a change in avoidances after surgery $[F(3,17)=3.72, p<.05]$. Specifically, preoperatively the high avoiders (Groups HS and HN) had an average of 49 avoidances, while the low avoiders (Groups LS and LN) averaged 11 avoidances. Postoperatively, Group LS had the largest increase (an average of 16 avoidances over 30 days) $[\mathrm{F}(1,17)=16.14, \mathrm{p}<.001]$, while Group HS had an increase of 10 avoidances $[F(1,17)=5.22, p<.05]$. Group LN had a nonsignificant postoperative decrease of three avoidances $[\mathrm{F}(1,17)=.62]$, while Group $\mathrm{HN}$ had a nonsignificant increase of five avoidances $[F(1,17)$ $=1.31 \mathrm{~J}$. However, if the last 5 preoperative and postoperative days (since these are the most stable days) are analyzed separately, Group HS showed an average increase of four avoidances, which was not significant $(p>.05)$. The major effect of septal lesions was an increase in avoidance in Group LS. Four out of six animals in this group exhibited the postoperative avoidance behavior summarized in Figure 2; however, two animals had slightly different postoperative trends. One animal did not show the characteristic decrease in avoidances after the fourth day-block, but maintained a high number of avoidances (58-60) throughout the 15 postoperative day-blocks. This animal was described in the histological results section as having the ventrally placed lesion. The other animal showed the postoperative increase in avoiding for the first four dayblocks, which then gradually declined to zero avoidances (preoperative average over 30 days) on the eighth postoperative day-block for the remaining test days. Histological examination revealed this to be the largest lesion (see Figure 1).

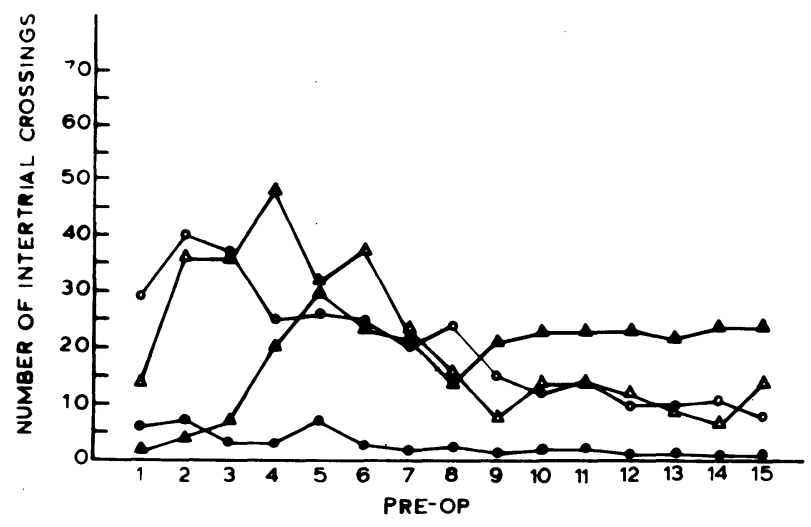

BLOCKS OF 2 DAYS $1.52]$.
There was also a significant Days by Surgery in teraction, indicating a large preoperative increase for all groups in avoidances from the first to the second dayblock $[F(14,238)=1.82, p<.05]$, while there was no difference in avoidances over postoperative days for all groups $[F(14,238)=1.41]$. The Group by Days by Surgery interaction was not significant $[F(42,238)=$

Figure 3 shows the mean number of intertrial crossings before and after surgery for the four groups of animals. The day effect revealed that the number of intertrial crossings decreased over days, with Day-Block 2 being the highest (25 intertrial crossings a session) and Day-Block 14, the lowest (9 crossings) $[F(14,238)=7.62, p<.001]$. A significant Days by Groups interaction indicated that Groups HS, HN, and LS decreased in intertrial crossings over days, while Group LN increased in crossings until Day-Block 5 $[F(42,238)=4.43, p<.001]$. Specifically, Group HS had the largest number of intertrial crossings on the first day-block (48 intertrial crossings, $\mathrm{p}<.01)$, while Group LS, HN, and LN had 20, 11, and 11 crossings, respectively. There was a significant Days by Surgery effect $[F(14,238)=3.39, p<.001]$, since both Groups LS and HS had a large postoperative increase in intertrial crossings during the first day-block (see Figure 3). There was no significant Groups by Days by Surgery interaction for intertrial crossings.

Analysis of escape latency indicated that there was a significant decrease over days in the amount of shock an animal received before escaping $[F(14,238)=6.73$, $\mathrm{p}<.001]$. On Day-Block 1, the animals received an average of $1.3 \mathrm{sec}$ shock per trial, while they received an average of $.8 \mathrm{sec}$ shock the remaining days $(p<.01)$. There was a significant postoperative decrease in escape latency, $.93 \mathrm{sec}$ to $.79 \mathrm{sec}[\mathrm{F}(1,17)=10.68, \mathrm{p}<.005]$. Although the low-avoiding groups (LS and LN) had a mean escape latency of $1.12 \mathrm{sec}$ and the high avoiders (HS and HN) had a mean escape latency of .58 sec, there was no significant group effect $[F(3,17)=2.73]$. The

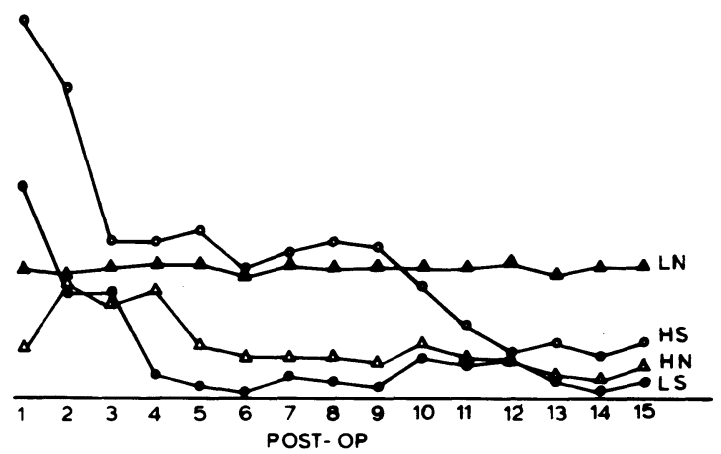

Figure 3. Mean number of intertrial crossings for low avoiders (LS) and high avoiders (HS) with septal lesions and low avoiders (LN) and high avoiders (HN) with control operations for each 60-trial session averaged in blocks of 2 days before and after surgery. 
analysis of avoidance latency revealed no significant main or interaction effects. All animals had an average avoidance latency of $5.4 \mathrm{sec}$.

\section{DISCUSSION}

In previous studies (Johnson et al., 1972; Kenyon \& Krieckhaus, 1965; Meyer et al., 1970; Schwartzbaum et al., 1967; Worsham \& Hamilton, 1973), complete septal lesions have resulted in a facilitated acquisition of an active avoidance response. In the present study, septal lesions caused an increase in avoidance behavior in animals with 1,800 preoperative trials of low avoiding. Postoperatively, low avoiders given septal lesions showed an increase in the number of avoidances on the first postoperative day. The number of postoperative avoidances was highest for this group between Day-Blocks 2 and 3 , subsequently declining from Day-Blocks 3 to 6, and then stabilizing from Day-Blocks 6 to 15. Group LS maintained a higher level of avoidance responding than Group LN throughout the postoperative testing period. The facilitatory effect of septal lesions on shuttlebox avoidance occurred in animals with few preoperative avoidances, while septal lesions did not change the avoidance behavior of the high avoiders.

D'Amato and Schiff (1964) found that in a long-term discriminated leverpress avoidance task there are generally two groups of animals, avoiders and nonavoiders. Even after extensive training, which totaled 7,330 trials, at least $50 \%$ of all rats tested failed to improve avoidance responding. This study reports a similar finding in a shuttlebox discriminated avoidance task. Of the unoperated rats before surgery, about half failed to improve avoidance responding, with an average of $18 \%$ avoidances.

Other studies (Johnson et al., 1972; Kenyon \& Krieckhaus, 1965; Meyer et al., 1970) showed that if intertrial crossings were punished, rats with septal lesions still exhibited an increase in the acquisition of an avoidance response. If intertrial crossings were not punished, rats with septal lesions showed significantly more crossings than controls (Schwartzbaum et al., 1967). Meyer et al. (1970) proposed that intertrial crossings were not solely responsible for the faster avoidance acquisition of septal animals, since intertrial crossings decreased during the latter $50 \%$ of the trials to criterion. In the present study, septal lesions caused an increase in nonpunished intertrial crossings; however, this difference was only apparent during the early postoperative sessions. Rats sustaining septal lesions showed a decrease in intertrial crossings over postoperative days while still maintaining a high level of avoidances (especially Group HS). The increase in postshock activity does not appear to be a function of a decrease in the detection threshold for footshock (Lints \& Harvey, 1969; Lubar,
Brener, Deagle, Numan, \& Clemens, 1970). This finding demonstrated that intertrial crossings were not the sole cause of increased avoidances after septal lesions.

Van Atta and Thomas (1975) suggested that the differential performance on a reversal learning task of animals receiving septal lesions before or after acquisition of a position habit may be explained by a retention deficit. Therefore, in the present study, a possible explanation for the postoperative improvement of animals in Group LS is that after surgery these animals behave as animals with no preoperative experience, since their responding was so low preoperatively that any improvement would be significant after the lesion. However, if this were true, animals in Group HS would have as likely become lower avoiders, instead of maintaining their high level of postoperative avoidances. Memory of the signaled avoidance task was not destroyed or rendered inaccessible by the lesion.

Similar effects of septal lesions and cholinergic blocking agents have suggested that behavior following septal lesions may be explained by disruption of cholinergic transmission (Grossman, 1964; Hamilton \& Grossman, 1969). Ellen, Aitken, Sims, and Stahl (1975) proposed that the behavioral effect of septal lesions on DRL behavior could not be explained solely by alterations of central cholinergic brain mechanisms. Scopolamine interferes with response suppression and was shown to enhance the acquisition of two-way avoidance responding in mice (Oliverio, 1967, 1968) and rats (Suits \& Isaacson, 1968). However, Oliverio (1967) found that scopolamine disrupted a previously learned avoidance response, and only facilitated avoidances during the initial phases of acquisition. In view of Oliverio's (1968) results, the present experiment showed that scopolamine and septal lesions do not have equivalent effects, since septal lesions did not disrupt the performance of the high avoiders.

Most studies investigating the effects of septal lesions on avoidance learning concentrated on the short-term acquisition of avoidance responding using naive animals. The present study investigated the effects of septal lesions on the long-term maintenance of avoidance responding in rats with established histories of either high or low performances on an avoidance task. The major findings were: (1) Septal lesions have a facilitatory effect on avoidance responding in animals with a previous history of escaping rather than avoiding shock. Despite procedural differences, this finding agrees with Hedges et al. (1975), thus indicating that changes in avoidance behavior following septal lesions are both stable and reliable. (2) Animals with septal lesions maintain a high level of avoiding over an extended period of time. (3) Septal lesions result in a temporary increase in nonpunished intertrial crossings that decrease over days without a change in maintaining avoidances. (4) Septal lesions interfered with the suppression of an ineffective 
defense reaction (freezing) without interfering with the effective defense reaction (running). This finding agrees with Blatt (1976).

\section{REFERENCES}

BLATt, R. C. Facilitation and nonfacilitation of active avoidance behavior of rats with septal lesions in the shuttle box and running wheel. Journal of Comparative and Physiological Psychology, 1976, 90, 704-713.

Bolles, R. C. Species-specific defense reactions and avoidance learning. Psychological Review, 1970, 77, 32-48.

Carlton, P. L. Brain-acetylcholine and inhibition. In J. T. Tapp (Ed.), Reinforcement and behavior. New York: Academic Press, 1969.

D' Amato, M. R., \& SchifF, D. Long-term discriminated avoidance performance in the rat. Journal of Comparative and Physiological Psychology, 1964, 57, 123-126.

DE GROOT, J. The rat forebrain in stereotaxic coordinate. Amsterdam: Noord-Hollandstche Vitgevers Maatschappiz, 1959.

Ellen, P., Aitken, W. C., Sims, T., \& Stahl, J. M. Cholinergic blockade, septal lesions, and DRL performance in the rat. Journal of Comparative and Physiological Psychology, 1975, 89, 409-420.

Fried, P. A. Septum and behavior: A review. Psychological Bulletin, 1972, 78, 292-310.

Grossman, S. P. Effect of chemical stimulation of the septal area on motivation. Journal of Comparative and Physiological Psychology, 1964, 58, 194-200.

Hamilton, L. W., \& Grossman, S. P. Behavioral changes following disruption of central cholinergic pathways. Journal of Comparative and Physiological Psychology, 1969, 69, 76-82.

Hedges, A. S., Van Atta, L., \& Thomas, J. B. Septal lesions facilitate the shift from conditioned escape to conditioned avoidance behavior in the rat. Physiology \& Behavior, 1975, 14, 25-30.

Johnson, D. A., Poplawsky, A., Bieliauskas, L., \& LIEBERT, D. Recovery of function on a two-way conditioned avoidance task following septal lesions in infancy: Effects of early handling. Brain Research, 1972, 45, 282-287.

Kenyon, J., \& KrieckHaus, E. E. Enhanced avoidance behavior following septal lesions in the rat as a function of lesion size and spontaneous activity. Journal of Comparative and Physiological Psychology, 1965, 59, 466-469.

Lints, C. E., \& HaRvey, J. A. Altered sensitivity to foot shock and decreased brain content of serotonin following brain lesions in the rat. Journal of Comparative and Physiological Psychology, 1969, 67, 23-31.

Lubar, J. F., Brener, J. M., Deagle, J. H., Numan, R., \& Clemens, W. J. Effect of septal lesions on detection threshold and unconditioned response to shock. Physiology \& Behavior, 1970, 5, 459-463.

MCCleary, R. A. Response specificity in the behavioral effects of limbic lesions in the cat. Journal of Comparative and Physiological Psychology, 1961, 54, 605-613.

MeYer, P. M., Johnson, D. A., \& VAughn, D. W. The consequences of septal and neocortical ablations upon learning a two-way conditioned avoidance response. Brain Research, 1970, 22, 113-120.

Oliverio, A. Contrasting effects of scopolamine on mice trained simultaneously with two different schedules of avoidance conditioning. Psychopharmacologia, 1967, 11, 39-51.

OLIVERIO, A. Effects of scopolamine on avoidance conditioning and habituation of mice. Psychopharmacologia, 1968, 12, 214-226.

Pellegrino, L. J., \& Cushman, A. J. A stereotaxic atlas of the rat brain. New York: Appleton-Century-Crofts, 1967.

Schwartzbaum, J. S., Green, R. H. Beatty, W. W., \& Thompson, J. B. Acquisition of avoidance behavior following septal lesions in the rat. Journal of Comparative and Physiological Psychology, 1967, 63, 95-104.

Suirs, E., \& IsaAcson, R. L. The effects of scopolamine hydrobromide on one-way and two-way avoidance learning in rats. International Journal of Neuropharmacology, 1968, 7, 1-6.

Van AtTA, L., \& Thomas, J. B. Interactive effects of training condition and septal lesions on perseverative responding in the rat. Journal of Comparative and Physiological Psychology, 1975, 89, 421-426.

Worsham, E., \& Hamilton, L. W. Acquisition and retention of avoidance behaviors following septal lesions or scopolamine injections in the rat. Physiological Psychology, 1973, 1, 219-226.

(Received for publication February 27, 1978; revision accepted June 2, 1978.) 